\title{
Controlador PID con algoritmos genéticos de números reales
}

Guillermo TeJada MuñoZ ${ }^{1}$

\begin{abstract}
RESUMEN
Un algoritmo genético o genetic algorithm (GA) es una técnica de la inteligencia artificial que es utilizada en cualquiera de las especialidades de ingeniería. En el presente estudio, el GA propuesto encuentra valores óptimos para los parámetros $\mathrm{Kp}$, Ki y $\mathrm{Kd}$ de un controlador PID, utilizado ampliamente en la industria. Los cromosomas, formados con los genes Kp, Ki y $\mathrm{Kd}$, representados por números reales, evolucionan y son evaluados mediante el error cuadrático medio (ECM) de la salida deseada. En ese sentido, la solución es el cromosoma con menor ECM, el cual produce menores transitorios en la salida. Además, el GA ha sido codificado en lenguaje M (MATLAB) y los resultados han sido comparados con otros trabajos.
\end{abstract}

Palabras-claves: Algoritmos genéticos; genes de números reales; PID; error cuadrático medio.

\section{INTRODUCCIÓN}

Un proceso industrial puede verse como un sistema cerrado (retroalimentado) constituido por un controlador y una planta controlada. Para el diseño del controlador, una de las técnicas más utilizadas, por su simplicidad y robustez, es la denominada proporcional integral derivativo (PID); sin embargo, no es una tarea fácil encontrar los valores óptimos de los parámetros del controlador denominados $\mathrm{Kp}$, Ki y Kd. Por ese motivo, para el cálculo de esos parámetros, se ha propuesto como solución utilizar algoritmos de inteligencia artificial; por ejemplo, se tiene: optimización de enjambre de partículas (PSO) (Iwan et al., 2011), optimización de colonia de hormigas (ACO) (Lahlouh et al., 2019), lógica difusa (Akbari-Hasanjani et al., 2015), redes neuronales (Hernández et al., 2016), algoritmos genéticos (GA) (Feng et al., 2018), entre otros. En el presente estudio se describe la elaboración de un GA, codificado en lenguaje M de MAT$\mathrm{LAB}$, que calcula los parámetros $\mathrm{Kp}$, Ki y Kd del controlador, de tal modo que la salida del sistema (controlador más planta) se estabilice al valor deseado, minimizando sus valores transitorios de sobreimpulso, tiempo de establecimiento y tiempo de subida.

Un algoritmo genético (GA) es un algoritmo de búsqueda que sigue el paradigma de la evolución. A partir de una población inicial, el algoritmo aplica operadores genéticos (selección, cruce y mutación) para producir descendientes (en la terminología de búsqueda local, corresponde a explorar el vecindario), que son claramente más aptos que sus antepasados. En cada generación (iteración), cada nuevo cromosoma corresponde a una solución (Pezzella et al., 2008). Los métodos para seleccionar los cromosomas que van a generar descendientes dan preferencia a aquellos con mayores valores de aptitud; entre estos métodos de selección se tiene a los siguientes: selección por ruleta, selección por torneo, ranking lineal, etc. Asimismo, en este estudio

Ingeniero electrónico y doctor en Ingeniería Industrial por la Universidad Nacional Mayor de San Marcos. Además, es magíster en Ingeniería Electrónica por la Universidad de São Paulo (Brasil). Actualmente, es profesor principal de la Facultad de Ingeniería Electrónica y Eléctrica de la UNMSM.

E-mail: gtejadam@unmsm.edu.pe 
se ha utilizado la selección de ranking lineal porque con ella se logra asignar a los cromosomas, ranqueados de acuerdo a su valor de aptitud, distintos valores de probabilidad que se diferencian en una proporción lineal decreciente desde el mejor hasta el peor ranqueado; es decir, las probabilidades que tienen los cromosomas de ser seleccionados no se diferencian en forma desproporcionada.

Los métodos para realizar el cruce de los cromosomas son diversos y dependen de la naturaleza de los genes, los cuales puede ser binarios, permutados, de números reales, etc. Así, entre los métodos de cruce, se tiene a los siguientes: SPX (single point exchange), DPX (double point crossover), PMX (partially matched crossover), OX (order crossover), CX (cycle crossover), aritmético, etc. (Haupt y Haupt, 2004). Este último, el aritméti$\mathrm{co}$, ha sido utilizado en el presente trabajo, por ser adecuado para genes de números reales. Así, mientras que el cruce tiende a que la población genética converja, preferiblemente a valores óptimos, la mutación tiene como objetivo mantener un cierto nivel de diversidad en la población, es decir, evitar que la población converja rápidamente (Srinivas y Patnaik, 1994; Schaffer y Eshelman, 1991; Beck, 2000). La mutación utilizada con mayor frecuencia es aquella en que uno de los genes (variables) del cromosoma varía aleatoriamente (Gestal et al., 2010). Por último, el número de mutaciones se calcula mediante un porcentaje con respecto al tamaño de la población.

De esta forma, el problema por solucionar es multiobjetivo, porque se desea minimizar más de una variable, las que son: el sobreimpulso, el tiempo de establecimiento y el tiempo de subida de la respuesta del sistema. No obstante, para mayor sencillez, el problema ha sido tratado como mono-objetivo, es decir, persiguiendo un solo objetivo, que se correlacione con las variables de interés. En este sentido, se ha seleccionado como función objetivo, también denominado como función aptitud, función de ajuste, función fitness o función costo, al error cuadrático medio (ECM) de la salida.

Por otro lado, el código del programa se ha escrito en lenguaje de MATLAB. El programa ha corrido controlando las plantas utilizados por otros investigadores, de esta forma se ha podido comparar resultados y demostrar que el algoritmo propuesto ha tenido un buen desempeño, ya que ha encontrado los valores adecuados de $\mathrm{Kp}$, Ki y Kd del controlador PID con los cuales se han minimizado el sobreimpulso, el tiempo de establecimiento y el tiempo de subida de la respuesta del sistema.

\section{METODOLOGÍA}

La estructura del algoritmo implementado se describe en la figura 1. Después de creada una población genética inicial, se evalúa cada cromosoma de la población calculando el error cuadrático medio (ECM) de la salida que le corresponde. Luego, se ordenan a los cromosomas de menor a mayor valor según su ECM y, posteriormente, entre los cromosomas más aptos se seleccionan a los que se van a cruzar con el objeto de generar descendencia, se mutan aleatoriamente a los cromosomas para luego realizar una nueva evaluación. El proceso se reitera tantas veces como lo permita el número de generaciones indicado por el usuario. Finalmente, se obtendrá la población óptima y el mejor cromosoma será el primero de la lista. En los párrafos siguientes se dan mayores precisiones sobre cada etapa.

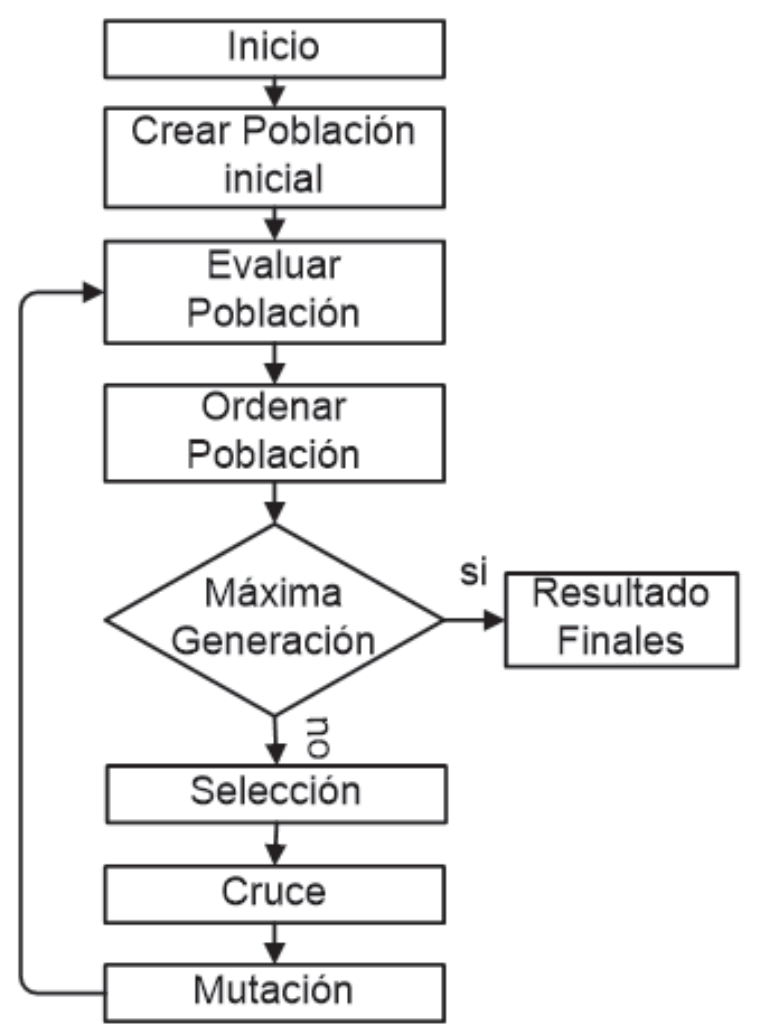

Figura 1. Estructura del algoritmo genético. Fuente: elaboración propia.

\section{Población}

La población genética está constituida por un número de cromosomas, en donde cada uno contiene un número de genes. Por la naturaleza del problema, se definió que cada cromosoma estuviera constituido por tres genes o variables de números reales, lo 
cual matemáticamente significa que un cromosoma es un vector de tres elementos, en donde cada elemento son las variables proporcional (KP), integral (Ki) y derivativa $(\mathrm{Kd})$. De esta manera, cada vector (cromosoma) es una probable solución del problema, por lo que la población genética se ha construido como un arreglo de $\mathrm{N}$ filas por tres columnas, donde $\mathrm{N}$ es el número de cromosomas o tamaño de la población y cada una de las tres columnas representan respectivamente a las variables $\mathrm{Kp}$, Ki y $\mathrm{Kd}$. La población inicial ha sido creada con números reales aleatorios respetando los intervalos mínimo y máximo (rangos) de cada variable indicadas por el usuario. La figura 2 muestra parte del código que crea a la población genética.

\section{Selección}

En el tipo de selección de ranking lineal, los cromosomas son clasificados de acuerdo con su valor de ajuste y un rango ri $\in\{1, \ldots, N\}$ se asigna a cada uno, donde $\mathrm{N}$ es el tamaño de la población. El mejor cromosoma obtiene el rango $\mathrm{N}$ mientras que el peor obtiene el rango 1. Luego: $\mathrm{Pi}=(2 \mathrm{ri}) / \mathrm{N}(\mathrm{N}+1)$ es la probabilidad de elegir el i-enésimo cromosoma en el ranking lineal (Pezzella et al., 2008).

En este sentido, se escogen a los $\mathrm{n}$ primeros cromosomas para cruzarse, donde $n$ es el producto de la tasa de cruce por el número total de la población. Luego, se le otorga mayor probabilidad de cruce a los mejores ranqueados, formando un arreglo denominado «ánfora», en donde los números que identifican a los cromosomas se repiten proporcionalmente a su importancia (Tejada, 2017). Así, por ejemplo, suponiendo que se ha escogido a los cuatro primeros cromosomas de la población $(1,2,3,4)$, con ellos se genera una «ánfora», dando mayor prioridad de ser elegido al primer cromosoma porque es el mejor ranqueado de la población; esta se construye de la siguiente manera:

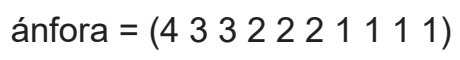

Esto indica que, al seleccionar aleatoriamente un cromosoma de la «ánfora», el cromosoma 1 tiene la mayor probabilidad de ser elegido $(4 / 10=40 \%)$, seguido del cromosoma $2(3 / 10=30 \%)$, luego el cromosoma $3(2 / 10=20 \%) y$, finalmente, el cromosoma $4(1 / 10=10 \%)$. Después, se selecciona de la «ánfora», mediante un sorteo, los padres y madres que se aparearán de acuerdo con el número de cruces especificado por el usuario. Se forman dos vectores, un vector de «padres» y un vector de «madres», así como se representan a continuación:

padres $=\left(\begin{array}{lllllll}1 & 2 & 1 & 2 & 2 & 1 & 1\end{array}\right)$

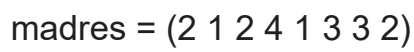

Hasta aquí, la etapa de selección termina su tarea. Luego, en la etapa de cruce, se van a cruzar los cromosomas: $(1,2),(2,1), \ldots,(1,3),(2,2)$. Los mismos padres pueden cruzarse varias veces y siempre van a generar hijos diferentes. Además, puede ocurrir que, tanto el padre como la madre, sean los mismos cromosomas, por lo que no es necesario evitarlo, esto no ha afectado el desempeño del algoritmo. La figura 3 muestra parte del código de la selección.

\section{Cruce}

El operador de cruce aritmético de números reales puede generar hasta dos descendientes que son una combinación lineal de dos cromosomas $X$ e $Y$ (Yalcinoz y Altun, 2001):

$$
\begin{aligned}
& \text { hijo } 1=\alpha X+(1-\alpha) Y \\
& \text { hijo } 2=(1-\alpha) X+\alpha Y
\end{aligned}
$$

Donde $\alpha$ se escoge aleatoriamente entre 0 y 1 .

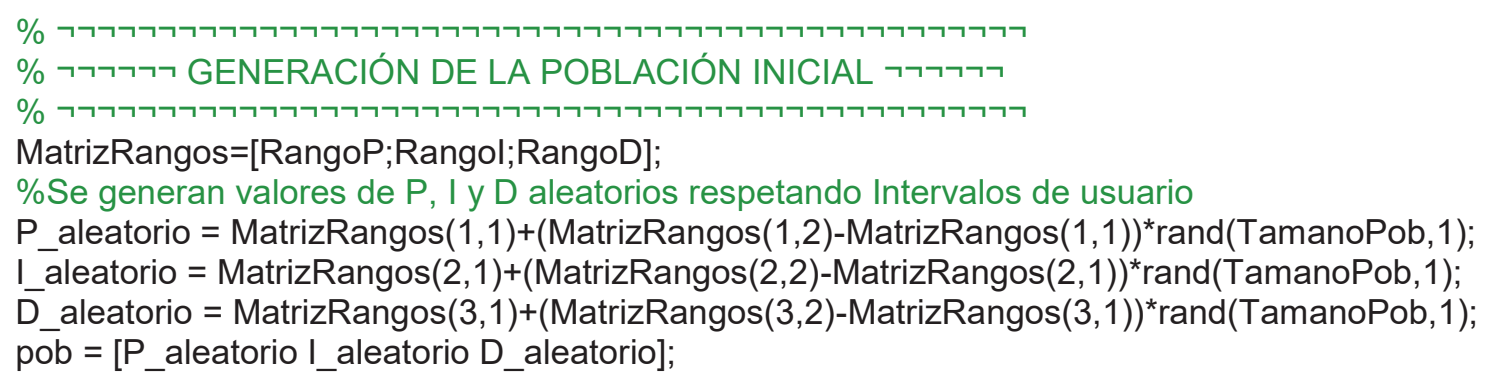

Figura 2. Parte del código que genera la población genética. 
En esta investigación se ha preferido generar por cada cruce un solo hijo, por lo que el número de padres es igual al número de cruces. Esto se ha realizado con el objeto de dar oportunidad a que más padres generen descendencia obteniendo mayor diversidad en la población. Si se hubiera optado por generar dos hijos en cada cruce, solo se hubiera seleccionado para cruzarse la mitad del número de padres. La figura 4 muestra parte del código de cruce.

\section{Mutación}

Una vez seleccionado al azar un cromosoma de la población y la variable que será modificada $(\mathrm{Kp}, \mathrm{Ki}$ o Kd), esta variable es remplazada por un número aleatorio:

pob(cromosoma, variable $) \leftarrow$ valor aleatorio

Sin embargo, como cada variable tiene un valor dentro de un intervalo especificado por el usuario (Imin, Imax); entonces, para evitar que la mutación destruya un valor válido de la variable, el valor aleatorio tiene que estar dentro de ese rango especificado, por lo que el reemplazo debe ser (Seck Tuoh, et al., 2016):

pob $($ cromosoma, variable $) \leftarrow \operatorname{Imin}+(\operatorname{Imax}-I \min )^{*}$ valor aleatorio

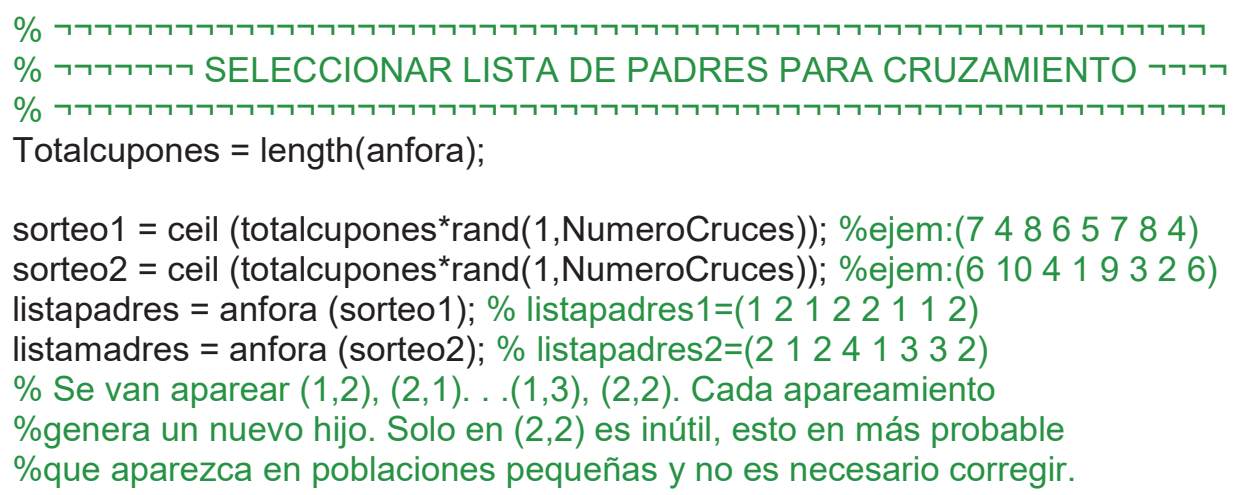

Figura 3. Parte del código de la selección.

Fuente: elaboración propia.

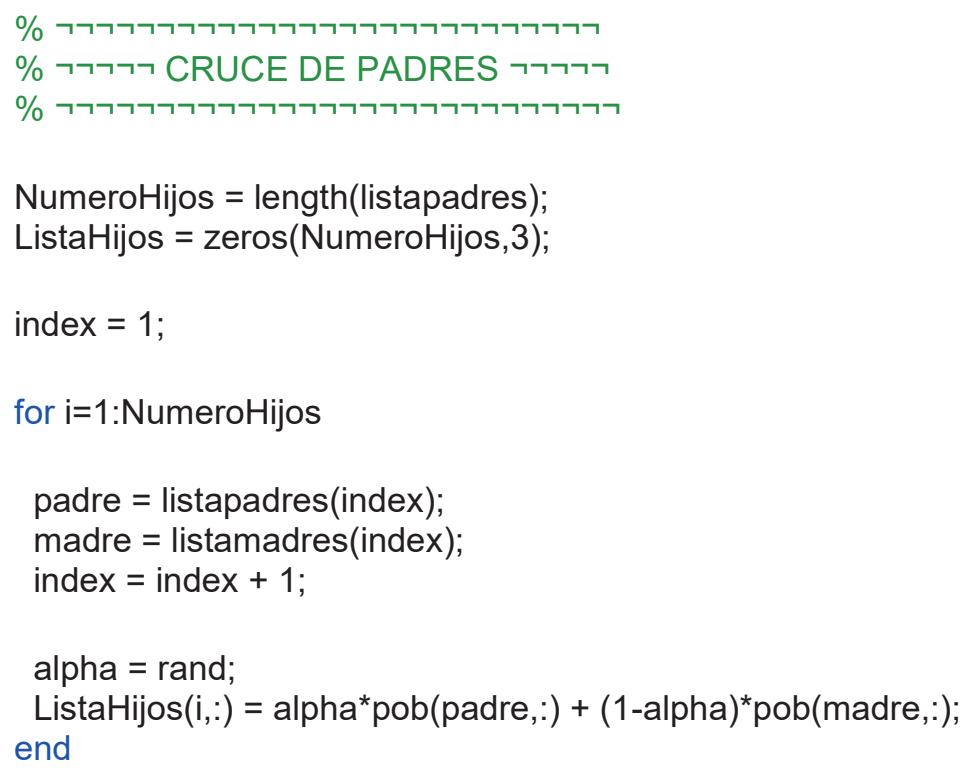

Figura 4. Parte del código de cruce. Fuente: elaboración propia. 
Otro importante punto que se ha tenido en cuenta es que nunca se elegirá al primer cromosoma para ser mutado, de tal manera que se conservará sin cambios al mejor rankeado, que pasará a la siguiente generación. La figura 5 muestra parte del código de mutación.

\section{Evaluación}

Se ha creado la función PID_ERROR_OBJETIVO, con la cual se evalúa a cada cromosoma de la población. De esta manera, con los valores $\mathrm{Kp}$, $\mathrm{Ki}$ y $\mathrm{Kd}$ de cada cromosoma se calcula la función de transferencia del controlador PID. Luego, con la función de transferencia del controlador y con la función de transferencia de la planta del sistema se configura un sistema cerrado retroalimentado. Finalmente, con el sistema cerrado retroalimentado se calcula su respuesta frente a la entrada de un escalón de amplitud uno (1). En todo este proceso, para abreviar cálculos, se han utilizado las funciones de MATLAB: tf (la cual crea una función de transferencia de tiempo continuo), series (la que conecta dos modelos o dos funciones de transferencias) y feedback (encuentra la función de dos modelos, uno de los cuales pertenece al lazo de realimentación), un procedimiento similar se realiza en el estudio de Ibrahim Mohamed (2005).

Durante el transitorio que demora la función de salida hasta alcanzar el valor de 1 , se hubiera podido encontrar por cada cromosoma los valores que toman las siguientes variables: tiempo de subida, sobreimpulso, tiempo de establecimiento, entre otros. De esta manera, se hubiera tenido que clasificar a los cromosomas que tengan a esas variables minimizadas, con lo cual el problema hubiera sido multiobjetivo. Sin embargo, todas las variables anteriores se pueden correlacionar con una sola variable, convirtiendo el problema a mono-objetivo. Es por eso que, en este estudio, se ha optado por calcular por cada cromosoma el error cuadrático medio (ECM) de la salida.

En ese sentido, el progreso del valor de la salida (Yi) hasta alcanzar el valor del escalón es leído durante $n$ espacios de tiempo, calculando el error cuadrático medio (ECM) de la siguiente forma:

$$
\mathrm{ECM}=\frac{1}{\mathrm{n}} \sum_{1}^{\mathrm{n}}(\mathrm{Yi}-1)^{2}
$$

Donde:

$\mathrm{n}$ = número de medidas totales

$\mathrm{Yi}=$ valor de la salida del sistema en el instante $\mathrm{i}$

$(\mathrm{Yi}-1)^{2}=$ error cuadrático de la salida en el instante i con respecto al escalón (1) de entrada.

Por cada cromosoma de la población, se tiene que realizar el cálculo de ECM de la manera antes descrita, con lo cual la función objetivo finaliza su tarea. Posteriormente, el algoritmo asocia a cada cromosoma un valor de ECM que lo identificará y los miembros de la población, de acuerdo con su ECM, son ordenados de menor a mayor valor, garantizando indirectamente un ranking ordenado de cromosomas de mejores características de tiempo de subida, sobreimpulso y tiempo de establecimiento. La figura 6 muestra la parte del código de evaluación.

El programa de software desarrollado se ha codificado en el lenguaje de MATLAB. Los resultados, tales como el valor de $\mathrm{Kp}, \mathrm{Ki}$, Kd y las características de la curva de salida, obtenidas con la función stepinfo, han sido exportados a una hoja de Excel mediante la función de MATLAB xlswrite, donde

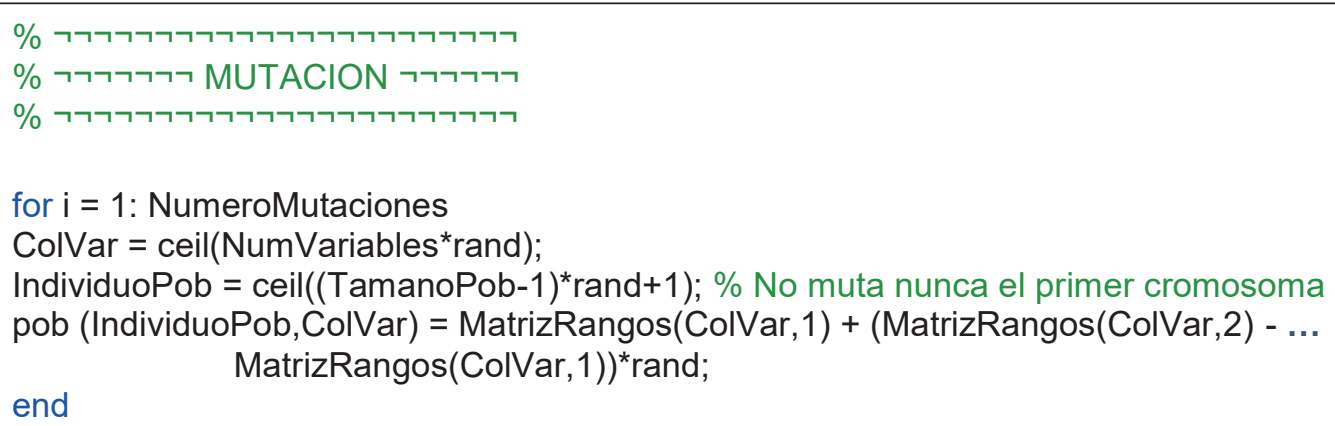

Figura 5. Parte del código de mutación.

Fuente: elaboración propia. 


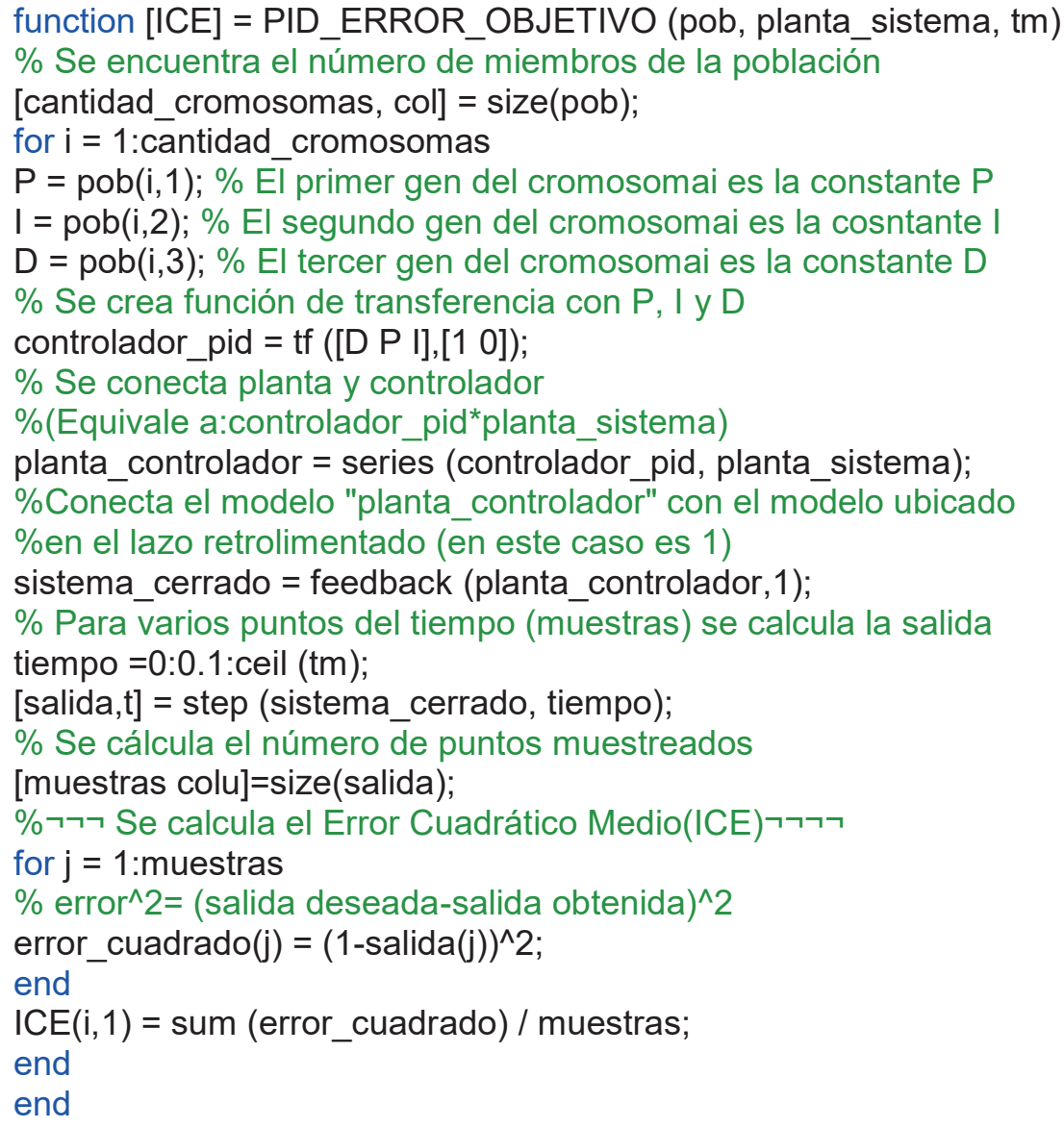

Figura 6. Código de evaluación.

Fuente: elaboración propia.

también se han generado los gráficos con las curvas de salida.

Los investigadores que han diseñado sus propios algoritmos genéticos o algoritmos genéticos difusos, entre otros, han contrastado sus resultados con los obtenidos con el método clásico de Ziegler-Nichols, exhibiendo buenos resultados. Por esta razón, se ha creído conveniente probar el algoritmo diseñado con los modelos de plantas utilizados por estos investigadores.

\section{RESULTADOS}

El programa ha sido ejecutado utilizando los modelos matemáticos de procesos (plantas) aplicados por otros estudiosos, lo cual ha permitido comparar resultados. Se ha seleccionado a los autores que han comparado sus datos con el método clásico de Ziegler-Nichols.
Para todos los casos, el programa se ha ejecutado con las siguientes especificaciones: tamaño de la población $=40$, número de variables $=3$, porcentaje de cruces $=70 \%$, porcentaje de mutación $=10 \%$, número de generaciones $=20$.

Las tablas 1-4 muestran el modelo matemático de la planta controlada, los parámetros del controlador PID (Kp, Ki y Kd), los valores transitorios de la salida (sobre pico máximo, tiempo de subida, tiempo de establecimiento) y el error cuadrático medio. Además, se comparan los resultados con los obtenidos por otros investigadores, la columna «Algoritmo Genético Propuesto» corresponde al presente trabajo. Los intervalos de búsqueda para cada variable se muestran en la última fila de cada tabla.

Las figuras 7-10 muestran gráficamente los resultados de las tablas anteriores, la salida deseada del sistema se muestra con líneas punteadas y ha sido fijada para una amplitud de 1 (un escalón Step Res- 
Tabla 1. Comparaciones con resultados para Planta 1.

\begin{tabular}{|c|c|c|}
\hline $\begin{array}{l}\text { Planta } 1 \\
\frac{5 S+2}{s^{3}+5 s^{2}+8 s+4}\end{array}$ & $\begin{array}{c}\text { Algoritmo Genético Pareto } \\
\text { (Acevedo et al., 2014) } \\
\text { Multivariable }\end{array}$ & $\begin{array}{c}\text { Algoritmo Genético } \\
\text { PROPUESTO }\end{array}$ \\
\hline Kp & 37,6692 & 45,4248 \\
\hline $\mathrm{Ki}$ & 18,9251 & 19,8057 \\
\hline $\mathrm{Kd}$ & 13,0447 & 7,3211 \\
\hline Sobre pico máximo (\%) & 0,9995 & 1,2674 \\
\hline Tiempo de subida (s) & No indica & 0,0545 \\
\hline Tiempo de establecimiento (s) & 1,14 & 0,0847 \\
\hline Error cuadrático medio & ------------------ & 0,0100 \\
\hline
\end{tabular}

Fuente: elaboración propia.

Tabla 2. Comparaciones con resultados para Planta 2.

\begin{tabular}{|c|c|c|}
\hline $\begin{array}{l}\text { Planta } 2 \\
\frac{10}{s^{2}+2 s+10}\end{array}$ & $\begin{array}{l}\text { Algoritmo Genético Pareto } \\
\text { (Acevedo et al., 2014) } \\
\text { Multivariable }\end{array}$ & $\begin{array}{l}\text { Algoritmo Genético } \\
\text { PROPUESTO }\end{array}$ \\
\hline Kp & 8,4047 & 48,1812 \\
\hline $\mathrm{Ki}$ & 17,1467 & 19,5887 \\
\hline Kd & 12,0918 & 11,6125 \\
\hline Sobre pico máximo (\%) & 1,0137 & 1,1786 \\
\hline Tiempo de subida (s) & No indica & 0,0180 \\
\hline Tiempo de establecimiento (s) & 1,9484 & 0,0291 \\
\hline Error cuadrático medio & 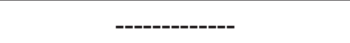 & 0,0099 \\
\hline
\end{tabular}

Fuente: elaboración propia.

Tabla 3. Comparaciones con resultados para Planta 3.

\begin{tabular}{|l|c|c|}
\hline $\begin{array}{c}1 \\
\text { Planta 3. Control de nivel de tres tanques }\end{array}$ & $\begin{array}{c}\text { Algoritmo Genético } \\
\text { (Colorado et al., 2018) }\end{array}$ & $\begin{array}{c}\text { Algoritmo Genético } \\
\text { PROPUESTO }\end{array}$ \\
\hline $64 s^{3}+9,6 s^{2}+0,48 s+0,008$ & 0,0361 & 0,03827 \\
\hline $\mathrm{Kp}$ & 0,000731 & 0,00061 \\
\hline $\mathrm{Ki}$ & 0,6999 & 0,63012 \\
\hline Kd & 18 & 12,3728 \\
\hline Sobre pico máximo (\%) & 10 & 16,3166 \\
\hline Tiempo de subida (seg.) & 90 & 86,0968 \\
\hline Tiempo de establecimiento (seg.) & 45 & 33,5201 \\
\hline Tiempo de pico & - & 0,03280 \\
\hline Error cuadrático medio & & \\
\hline Búsqueda: $0 \leq \mathrm{kp} \geq 0.5 ; 0 \leq \mathrm{ki} \geq 0.01 ; 0 \leq \mathrm{kd} \geq 0.7$ & & \\
\hline
\end{tabular}

Fuente: elaboración propia. 
Tabla 4. Comparaciones con resultados para Planta 4.

\begin{tabular}{|l|l|l|}
\hline $\begin{array}{l}\text { Planta 4. Control de nivel de tanque } \\
\frac{5(s+1)}{s(s+1)(s+6)}=\frac{5 s+5}{s^{3}+7 s^{2}+6 s}\end{array}$ & $\begin{array}{l}\text { Fuzzy PD+PID Controller (Souran et } \\
\text { al., 2013) }\end{array}$ & $\begin{array}{l}\text { Algoritmo Genético } \\
\text { PROPUESTO }\end{array}$ \\
\hline $\mathrm{Kp}$ & 30 & 39,10042 \\
\hline $\mathrm{Ki}$ & 21,2 & 1,16204 \\
\hline $\mathrm{Kd}$ & 9 & 6,86381 \\
\hline Sobre pico máximo (\%) & 0,72 & 0 \\
\hline Tiempo de subida (seg.) & No indica & 0,06548 \\
\hline Tiempo de establecimiento (seg.) & 23 & 0,12271 \\
\hline Error cuadrático medio & & 0,01236 \\
\hline Búsqueda: $0 \leq \mathrm{kp} \geq 40 ; 0 \leq \mathrm{ki} \geq 30 ; 0 \leq \mathrm{kd} \geq 20$ & \multicolumn{2}{l}{} \\
\hline
\end{tabular}

Fuente: elaboración propia.

Step Res ponse

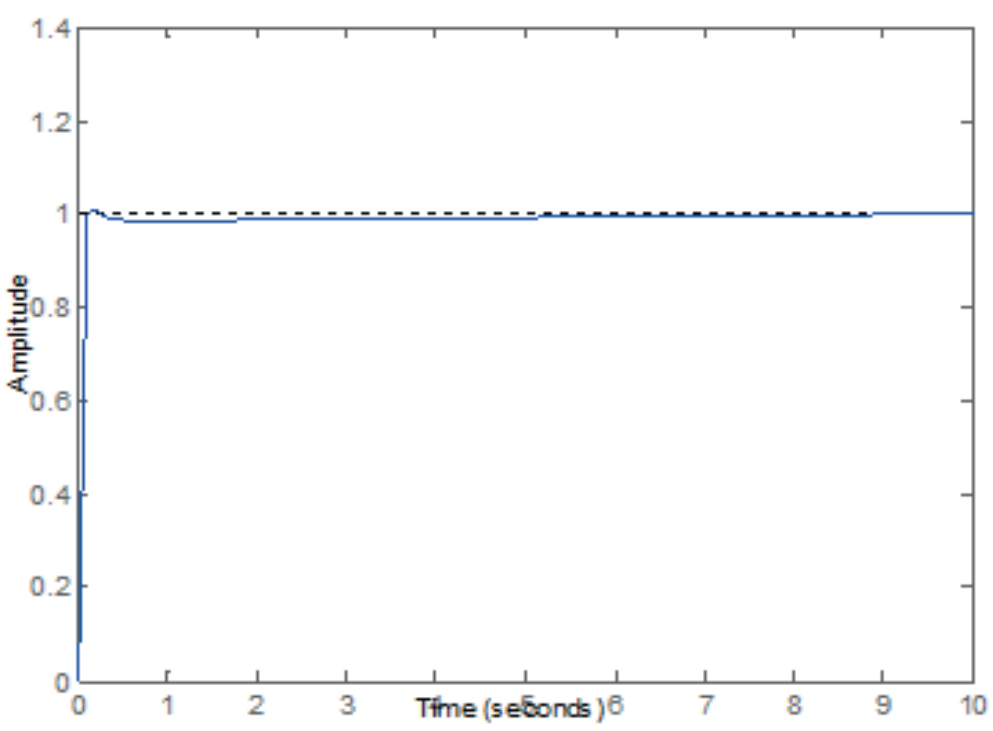

Figura 7. Respuesta controlada de la Planta 1 (tabla 1).

Fuente: elaboración propia.

ponse). Además, la línea azul es la salida del sistema que alcanza finalmente a la salida deseada.

\section{DISCUSIÓN}

Como se puede observar de los resultados de las tablas 1-4, en todos los casos los sobre picos máximos han sido similares, los tiempos de establecimientos han sido superiores en todos los casos y solo en la tabla 3 el tiempo de subida no ha sido el mejor. Estos resultados demuestran que el algoritmo propuesto ha tenido un buen desempeño en encontrar los valores adecuados de $\mathrm{Kp}$, Ki y Kd del controlador PID. Además, para todos los casos se ha utilizado las mismas especificaciones de tamaño de población, porcentaje de cruce, porcentaje de mutación y número de generaciones lo cual demuestra que el algoritmo es bastante robusto.

\section{CONCLUSIONES}

Mediante algoritmos genéticos, se ha solucionado el problema de sintonizar los parámetros proporcional, derivativo e integral de un controlador PID, 
Step Response

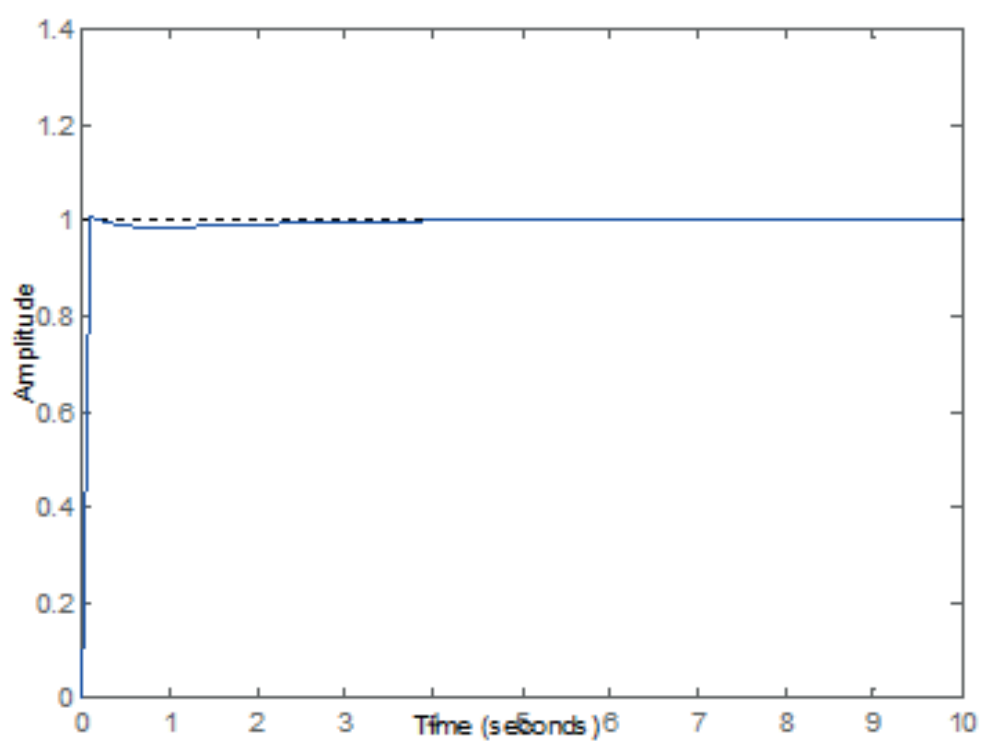

Figura 8. Respuesta controlada de la Planta 2 (tabla 2).

Fuente: elaboración propia.

Step Res ponse

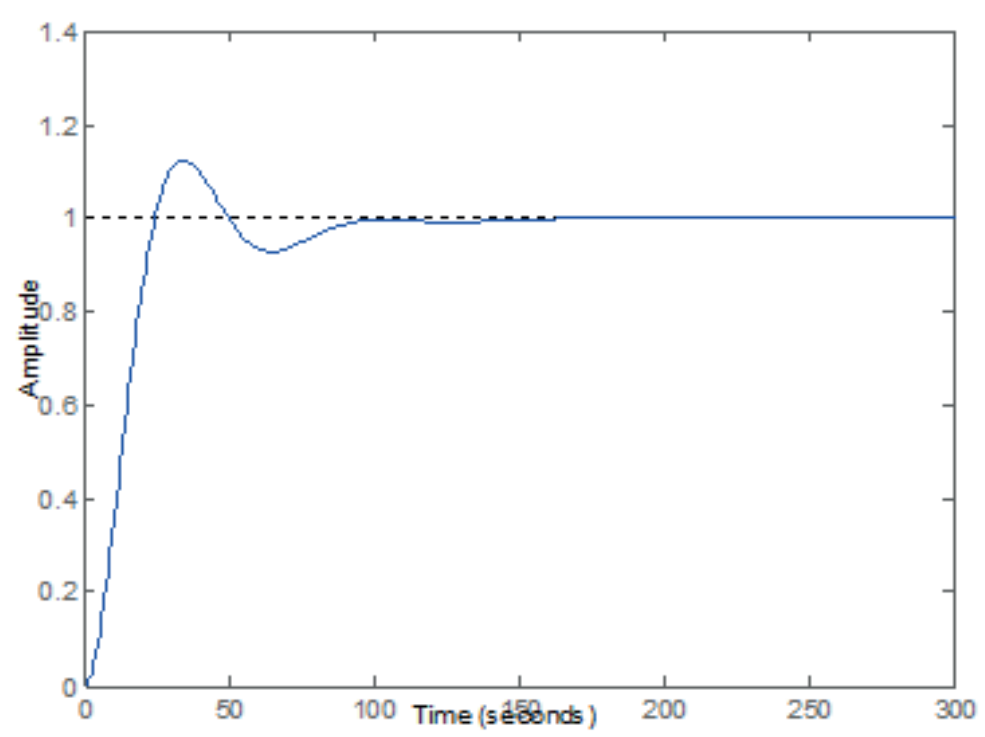

Figura 9. Respuesta controlada de la Planta 3 (tabla 3).

Fuente: elaboración propia. 


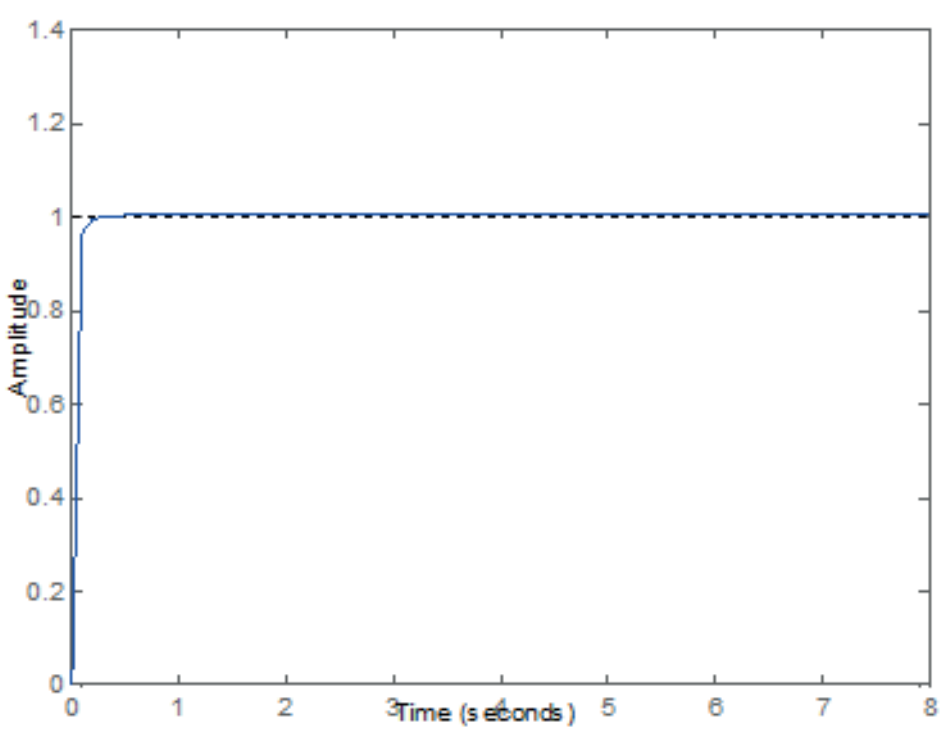

Figura 10. Respuesta controlada de la Planta 4 (tabla 4).

Fuente: elaboración propia.

minimizando el tiempo de establecimiento y el sobreimpulso de la respuesta en plantas no lineales. Asimismo, se ha probado que los resultados obtenidos han sido óptimos al compararlos con resultados de otras investigaciones, mostrados en las tablas 1-4.

Además, se ha probado que el algoritmo diseñado como mono-objetivo ha sido eficiente al minimizar, indirectamente, diversas variables (sobreimpulso, tiempo de establecimiento, tiempo de subida) mediante la minimización de una sola variable: el error cuadrático medio, en contraposición con un algoritmo genético multiobjetivo basado en el enfoque de Pareto, como el presentado por Acevedo et al. (2014).

\section{REFERENCIAS BIBLIOGRÁFICAS}

[1] Acevedo, B.; Fonseca, J. y Gómez, J. (2014). Desarrollo de una herramienta en Matlab para sintonización de controladores PID, utilizando algoritmos genéticos basado en técnicas de optimización multiobjetivo. Revista SENNOVA, 1(1), 80-103.

[2] Akbari-Hasanjani, R.; Javadi, S. y SabbaghiNadooshan, R. (2015). DC motor speed control by self-turning fuzzy PID algorithm. Transactions of the Institute of Measurement and Control, 37(2), 164-176.
[3] Beck, F. (2000). Escalonamiento de tarefas jobshop realistas utilizando algoritmos genéticos en Matlab. (Tesis de maestría). Universidad Federal de Santa Catarina, Florianópolis, SC.

[4] Colorado, O.; Hernández, N.; Seck Tuoh, J. y Medina, J. (2018). Algoritmo genético aplicado a la sintonización de un controlador PID para un sistema acoplado de tanques. PADI Boletín Científico de Ciencias Básicas e Ingenierías del ICBI, 5(10), 50-55.

[5] Feng, H.; Yin, C.; Weng, W.; Ma, W.; Zhou, J.; Jia, W. y Zhang, Z. (2018). Robotic excavator trajectory control using an improved GA based PID controller. Mechanical Systems and Signal Processing, 105, 153-168.

[6] Gestal, M.; Rivero, D.; Rabuñal, J.; Dorado, J. y Pazos, A. (2010). Introducción a los algoritmos genéticos y la programación genética. La Coruña, España: Universidad de La Coruña.

[7] Haupt, R. y Haupt, S. (2004). Practical genetic algorithms. Nueva Jersey, EE. UU.: WileyInterscience.

[8] Hernández, R.; García, L.; Salgado, T.; Gómez, A. y Fonseca, F. (2016). Neural network-based self-turning PID control for underwater vehicles. Sensors, 16(9). Recuperado de https://www. ncbi.nlm.nih.gov/pmc/articles/PMC5038707/. 
[9] Iwan, M.; Fook, L. y Leap, M. (2011). Tuning of PID controller using particle swarm optimization (PSO). International Journal on Advanced Science, Engineering and Information Technology, 1(4), 458-461. Recuperado de http://insightsociety.org/ojaseit/index.php/ ijaseit/article/viewFile/93/98.

[10] Lahlouh, I.; Elakkary, A. y Sefiani, N. (2019). PID controller of a MIMO system using ant colony algorithm and its application to a poultry house system. En H. Hachimi (Ed.), 2019 5th International Conference on Optimization and Application (ICOA) llevado a cabo en Marruecos.

[11] Mohamed, I. (2005). The PID controller design using genetic algorithm. (Tesis de pregrado). University of Southern Queensland, Toowoomba, QLD.

[12] Pezzella, F.; Morganti, G. y Ciaschetti, G. (2008). A genetic algorithm for the flexible job-shop scheduling problema. Computers \& Operations Research, 35(10), 3202-3212.

[13] Schaffer, J. y Eshelman, L. (1991). On crossover as an evolutionarily viable strategy. International Conference on Genetic Algorithms, 4, 61-68.
[14] Seck Tuoh, J.; Medina, J. y Hernández, N. (2016). Introducción a los algoritmos genéticos con Matlab. Recuperado de https:// repository.uaeh.edu.mx/bitstream/bitstream/ handle/123456789/16991/introduccion_a_los_ algoritmos_geneticos_con_matlab.pdf.

[15] Souran, D.; Abbasi, S. y Shabaninia, F. (2013). Comparative study between tank's water level control using PID and fuzzy logic controller. En V. Balas, J. Fodor, A. Várkonyi-Kóczy, J. Dombi y L. Jain (Eds.), Soft computing applications V. 195 (pp. 141-153). Berlín, Alemania: SpringerVerlag Berlin Heidelberg.

[16] Srinivas, M. y Patnaik, L. (1994). Adaptive probabilities of crossover and mutation in genetic algorithms. IEEE Transactions on Systems, Man, and Cybernetics, 24(4), 656667.

[17] Tejada, G. (2017). Enrutamiento y secuenciación óptimos en un flexible job shop multiobjetivo mediante algoritmos genéticos. (Tesis doctoral). Universidad Nacional Mayor de San Marcos, Lima.

[18] Yalcinoz, T. y Altun, H. (2001). Power economic dispatch using a hybrid genetic algorithm. IEEE Power Engineering Review, 21(3), 59-60. 


\section{PID Controller with Real Number Genetic Algorithms}

Guillermo Tejada Muñoz ${ }^{1}$

\section{ABSTRACT}

A genetic algorithm $(G A)$ is an artificial intelligence technique that can be applied to any engineering specialty. In this study, the proposed GA finds optimal values for PID controller parameters $\mathrm{Kp}, \mathrm{Ki}$ and $\mathrm{Kd}$, which are widely used in the industry. Chromosomes, composed of $\mathrm{Kp}, \mathrm{Ki}$ and $\mathrm{Kd}$ genes, represented by real numbers, evolve and are evaluated by the mean square error (MSE) of the desired output. In that sense, the solution is the chromosome with the lowest MSE, which produces less transient output. In addition, the GA has been encoded in MATLAB language and results have been compared with other works.

Keywords: Genetic algorithms; real number genes; PID; mean square error.

\section{INTRODUCTION}

An industrial process can be considered a closed-loop (feedback) system consisting of a controller and a controlled plant. For controller design, one of the most used techniques, due to its simplicity and robustness, is the so-called proportional integral derivative (PID); however, it is not an easy task to find the optimal values of controller parameters $\mathrm{Kp}, \mathrm{Ki}$ and $\mathrm{Kd}$. For this reason, artificial intelligence algorithms have been proposed as a solution for the calculation of these parameters, for example: particle swarm optimization (PSO) (Iwan et al., 2011), ant colony optimization (ACO) (Lahlouh et al., 2019), fuzzy logic (Akbari-Hasanjani et al., 2015), neural networks (Hernández et al., 2016) and genetic algorithms (GA) (Feng et al., 2018) among others. This study describes the development of a GA, encoded in MATLAB's M-language, that calculates the controller's $\mathrm{Kp}, \mathrm{Ki}$ and Kd parameters, so that the output of the system (controller plus plant) reaches the desired value, minimizing transient overshoot values, settling time and rise time.

A genetic algorithm (GA) is a search algorithm that follows the evolutionary paradigm. From an initial population, the algorithm applies genetic operators (selection, crossover and mutation) to produce offspring (in local search terminology, neighborhood search method), who are clearly fitter than their parents. In each generation (iteration), each new chromosome corresponds to a solution (Pezzella et al., 2008). The methods for selecting the chromosomes that will generate descendants prioritize those with higher fitness values; among these selection methods are the following: roulette wheel selection, tournament selection, linear ranking, etc. In this study, linear ranking selection was used because it makes it possible to assign the chromosomes, ranked according to their fitness value, probability values that differentiated in a decreasing linear proportion the best to the worst ranked; that is to say, the probabilities the chromosomes have of being selected are not differentiated disproportionately.

1 Electrical engineer and $\mathrm{PhD}$ in Industrial Engineering from the Universidad Nacional Mayor de San Marcos. Master in Electronic Engineering from the Universidade de São Paulo (Brazil). Currently working as Professor at the School of Electrical and Electronic Engineering of the UNMSM.

E-mail: gtejadam@unmsm.edu.pe 
The methods for crossing chromosomes are diverse and depend on the nature of the genes, which can be binary, permutation, real-number, etc. Among crossing methods are the following: single point exchange (SPX), double point crossover (DPX), partially matched crossover (PMX), order crossover (OX), cycle crossover (CX), arithmetic crossover, etc. (Haupt \& Haupt, 2004). This last one, the arithmetic crossover, has been used in this study, because it is suitable for real-number genes. Thus, while crossing tends to converge the genetic population, preferably at optimal values, mutation aims to maintain a certain level of diversity within the population, that is, to prevent the population from converging rapidly (Srinivas \& Patnaik, 1994; Schaffer \& Eshelman, 1991; Beck, 2000). The most frequently used mutation is one in which one of the chromosome genes (variables) randomly varies (Gestal et al., 2010). Lastly, the number of mutations is calculated as a percentage of population size.

Therefore, the problem to be solved is multi-objective, because the idea is to minimize more than one variable, which are: overshoot, settling time and rise time of the system response. However, for simplicity's sake, the problem has been treated as a mono-objective, that is, pursuing a single objective, which is correlated with the variables of interest. In this sense, the mean square error (MSE) of the output has been selected as the objective function, also called the fitness function, evaluation function or cost function.

On the other hand, the program code was written in MATLAB language. The program was run monitoring the plants used by other researchers. In this way it was possible to compare results and demonstrate that the proposed algorithm performed well, since it found the appropriate PID controller Kp, Ki and $\mathrm{Kd}$ values, which have minimized overshoot, settling time and rise time of the system response.

\section{METHODOLOGY}

The structure of the implemented algorithm is described in Figure 1. After creating an initial genetic population, each chromosome in the population is evaluated by calculating the mean square error (MSE) of the corresponding output. Next, the chromosomes are ordered from the lowest to the highest value according to their MSE and, subsequently, the most suitable chromosomes are selected to be crossed in order to generate offspring, randomly mutating the chromosomes for further evaluation. The process is repeated as many times as the number of generations indicated by the user permits.
Finally, the optimal population will be obtained and the best chromosome will be at the top of the list. Further details on each stage are provided in the following paragraphs.

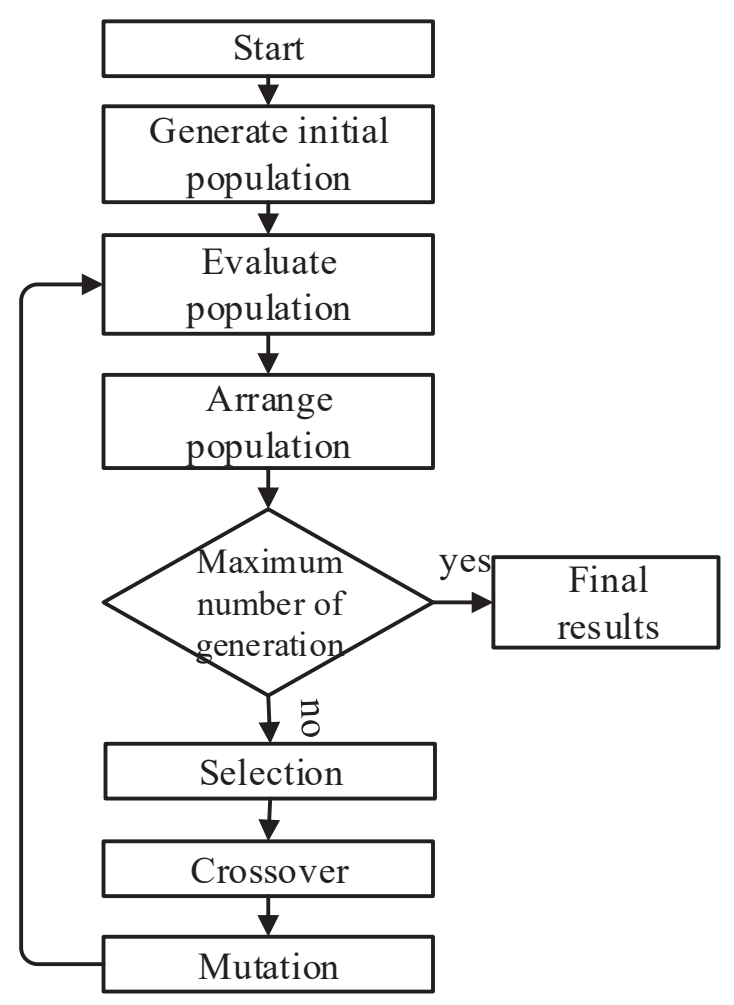

Figure 1. Genetic algorithm structure.

Source: Prepared by the author.

\section{Population}

The genetic population consists of a number of chromosomes, each containing a number of genes. Due to the nature of the problem, each chromosome was made up of three real-number genes or variables, which mathematically means that a chromosome is a vector of three elements, which are $\mathrm{Kp}$ (proportional), Ki (integral) and Kd (derivative) variables. Consequently, each vector (chromoso$\mathrm{me}$ ) is a likely solution to the problem, therefore the genetic population was built as an arrangement of $\mathrm{N}$ rows with three columns, where $\mathrm{N}$ is the number of chromosomes or population size and each of the three columns represent the variables $\mathrm{Kp}, \mathrm{Ki}$ and $\mathrm{Kd}$, respectively. The initial population was created with random real numbers respecting the minimum and maximum intervals (ranges) of each variable indicated by the user. Figure 2 shows a portion of the code that creates the genetic population. 


\section{Selection}

In linear ranking selection, chromosomes are classified according to their fitness value and a range $r i \in\{1, \ldots, N\}$ is assigned to each of them, where $N$ is the size of the population. The best chromosome obtains range $\mathrm{N}$ while the worst gets range 1 . Then: $\mathrm{Pi}=(2 \mathrm{ri}) / \mathrm{N}(\mathrm{N}+1)$ is the probability of choosing the i-nth chromosome in the linear ranking (Pezzella et al., 2008).

In this sense, the first $\mathrm{n}$ chromosomes are selected for crossover, where $\mathrm{n}$ is the product of the rate of crossing by the total population number. Then, greater probability of crossing is given to the best ranked, forming an arrangement referred to as "strand", where the numbers that identify the chromosomes are repeated proportionally to their importance (Tejada, 2017). For example, assuming that the first four chromosomes in the population have been chosen $(1,2,3,4)$, they generate a "strand", giving the first chromosome higher priority because it is the best ranked within the population. It is constructed as follows:

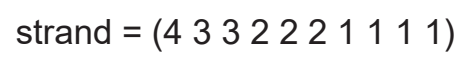

This indicates that, by randomly selecting a chromosome from the "strand", chromosome 1 is most likely to be chosen $(4 / 10=40 \%)$, followed by chromosome $2(3 / 10=30 \%)$, then chromosome $3(2 / 10=20 \%)$ and finally chromosome $4(1 / 10=10 \%)$. Next, from the "strand", the parents who will be mated according to the number of crossovers specified by the user, are randomly selected. Two vectors are formed, a "father" vector and a "mother" vector, as follows:

fathers $=\left(\begin{array}{lllllll}1 & 2 & 1 & 2 & 2 & 1 & 1\end{array}\right)$

mothers $=\left(\begin{array}{lllllll}2 & 1 & 2 & 4 & 1 & 3 & 3\end{array}\right)$

At this point, selection ends. Next, crossover begins, and the chromosomes to be crossed are: $(1,2),(2$,
$1), \ldots,(1,3),(2,2)$. The same parents can crossover several times and will always generate different offspring (children). In addition, it may happen that both the father and the mother are the same chromosomes; it is not necessary to avoid this, as it has not affected the performance of the algorithm. Figure 3 shows a portion of the selection code.

\section{Crossover}

An arithmetic crossover operator of real numbers may produce up to two offspring, which are a linear combination of two chromosomes $X$ and $Y$ (Yalcinoz \& Altun, 2001):

$$
\begin{aligned}
& \text { child } 1=\alpha X+(1-\alpha) Y \\
& \text { child } 2=(1-\alpha) X+\alpha Y
\end{aligned}
$$

where $\alpha$ is a random number between 0 and 1 .

In this study the preference was to generate one child per crossover, so the number of parents is equal to the number of crossovers. This has been done in order to give more parents the opportunity to generate offspring, obtaining greater diversity in the population. If it had been decided to produce two children at each crossover, only half of the number of parents would have been selected for crossover. Figure 4 shows a portion of the crossover code.

\section{Mutation}

Once a chromosome and variable to be modified $(\mathrm{Kp}, \mathrm{Ki}$ or $\mathrm{Kd}$ ) have been randomly selected from the population, as well as the variable to be modified ( $\mathrm{Kp}, \mathrm{Ki}$ or $\mathrm{Kd})$, the variable is replaced by a random number:

pop(chromosome, variable) random value

However, since each variable has a value within a user-specified range ( $\operatorname{minl}, \operatorname{maxl})$, the random value

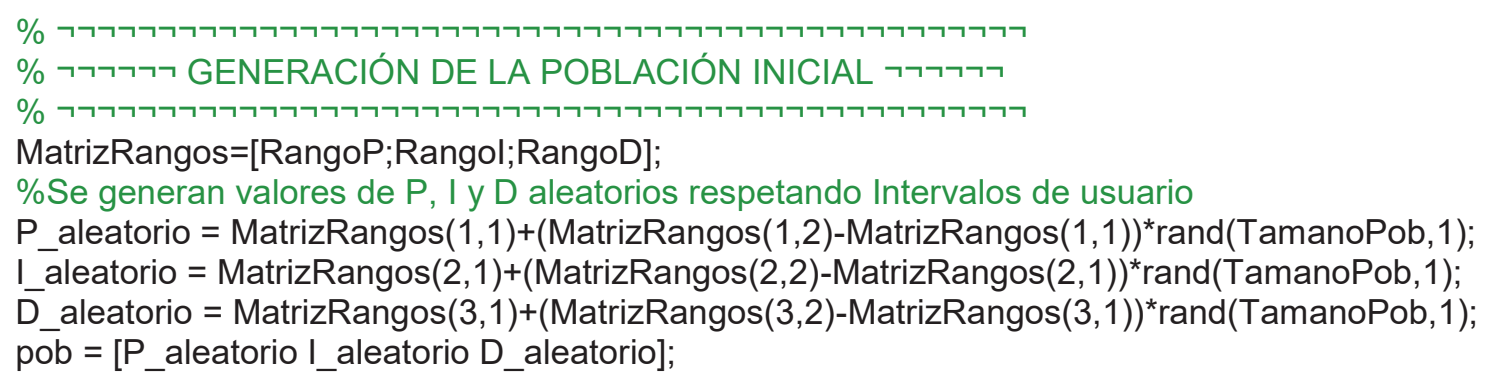

Figure 2. Portion of the code that generates the genetic population. 
has to be within that specified range in order to prevent mutation from destroying a valid value of the variable, thus the replacement must be (Seck Tuoh, et al., 2016):

pop $($ chromosome, variable $) \leftarrow \operatorname{minl}+$ (maxl-minl)*random value

Another important point that has been taken into account is that the first chromosome will never mutated so that the highest ranked chromosome, which will pass to the next generation, will remain unchanged. Figure 5 shows a portion of the mutation code.

\section{Evaluation}

Function PID_ERROR_OBJETIVO has been created, with which each chromosome in the population is evaluated. Thus, the transfer function of the PID controller is calculated using the $\mathrm{Kp}, \mathrm{Ki}$ and $\mathrm{Kd}$ values of each chromosome. Subsequently, with the controller transfer function and the system plant transfer function, a close-loop feedback system is configured. Finally, response to the input of a step of amplitude one (1) is calculated using the closed-loop system. The following MATLAB functions have been used to abbreviate calculations throughout the

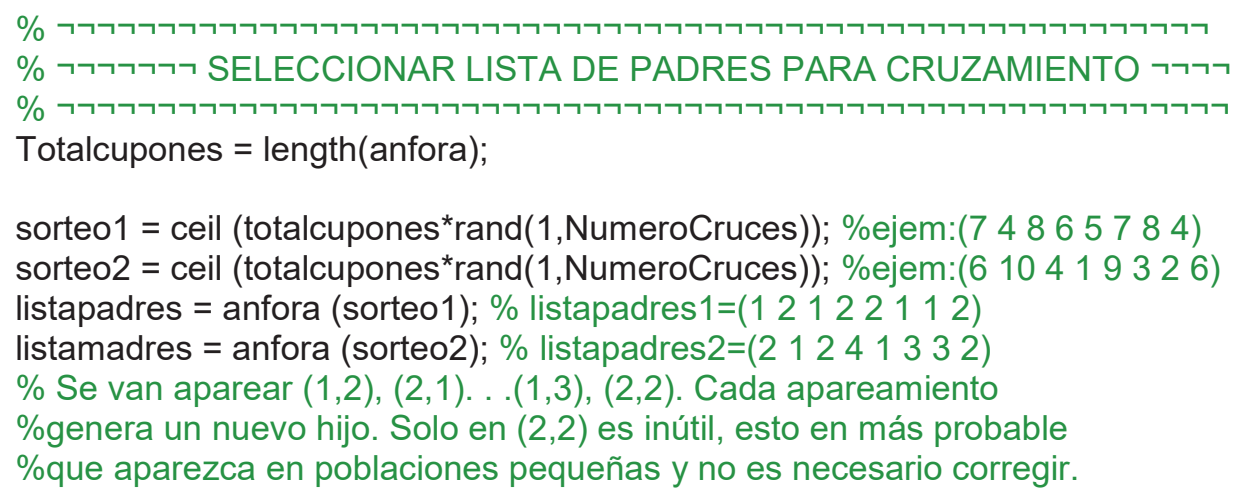

Figure 3. Portion of the selection code. Source: Prepared by the author.

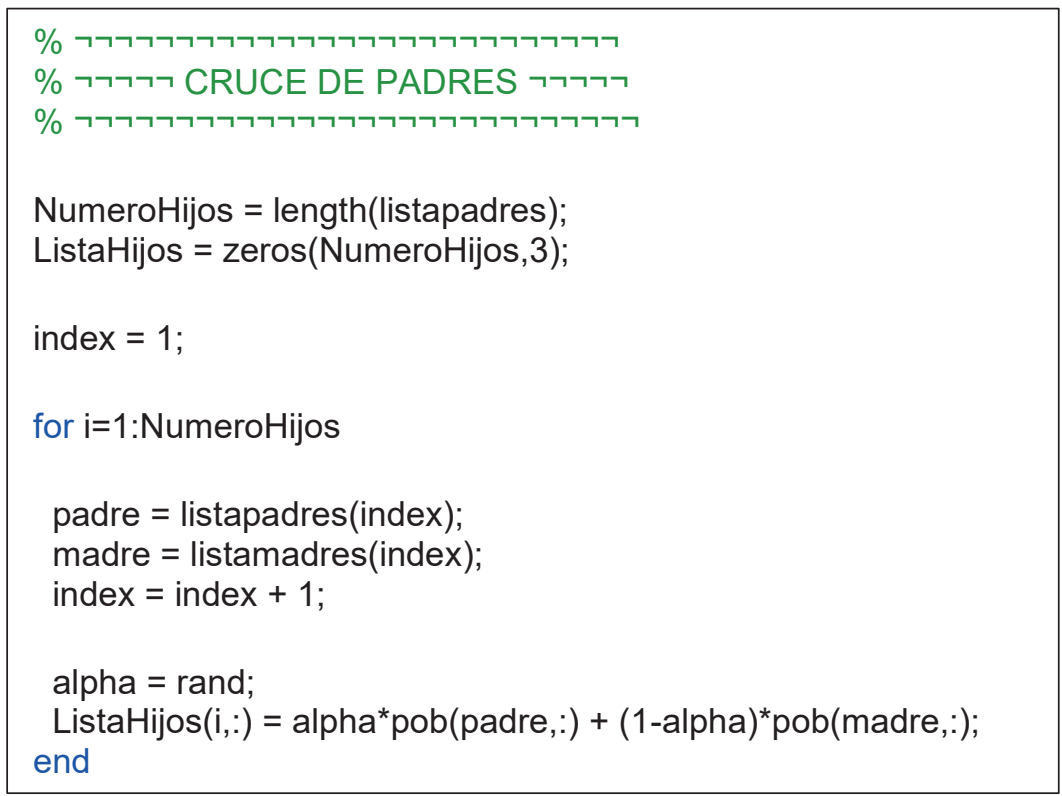

Figure 4. Portion of the crossover code.

Source: Prepared by the author. 
entire process: tf (which creates a continuous-time transfer function), series (which connects two models or two transfer functions) and feedback (finds the function of two models, one of which belongs to the feedback loop). A similar procedure is performed in the study by Ibrahim Mohamed (2005).

During the transient response time it takes the output function to reach a value of 1 , it would have been possible to find the values taken by the following variables, per chromosome, could have been found: rise time, overshoot, settling time, among others. Accordingly, chromosomes with minimized variables would have had to be classified, and as a result, the problem would have been multi-objective. However, all the above variables can be correlated with a single variable, producing a single-objective problem. For that reason, in this study, it was decided to calculate the mean square error (MSE) of the output per chromosome.

In this sense, the progress of the output value (Yi) until reaching the step value is read over $n$ intervals of time, calculating the mean square error (MSE) as follows:

$$
\mathrm{ECM}=\frac{1}{\mathrm{n}} \sum_{1}^{\mathrm{n}}(\mathrm{Yi}-1)^{2}
$$

Where:

$\mathrm{n}=$ total number of intervals

$\mathrm{Yi}=$ system output value at moment $\mathrm{i}$

$(Y i-1)^{2}=$ mean square error of the output at moment i regarding input step (1)

For each chromosome in the population, the MSE calculation has to be performed as described above, and thus the objective function completes its task. Subsequently, the algorithm associates a MSE value to each chromosome, which will identify it; the members of the population are ordered from lowest to highest value according to their MSE, indirectly guaranteeing an orderly ranking of chromosomes with better characteristics of rise time, overshoot and settling time. Figure 6 shows a portion of the evaluation code.

The software developed has been encoded in MAT$\mathrm{LAB}$ language. The results, such as $\mathrm{Kp}, \mathrm{Ki}, \mathrm{Kd}$ values and the output curve's characteristics obtained by means of stepinfo function, have been exported to an Excel sheet using the MATLAB xlswrite function, where output curves plots have also been generated.

Researchers who have designed their own genetic algorithms or fuzzy genetic algorithms, among others, have contrasted their results with those obtained with the classic Ziegler-Nichols method, obtaining good results. For this reason, it was considered appropriate to test the algorithm designed with the plant models used by these researchers.

\section{RESULTS}

The program has been executed using the mathematical models of processes (plants) applied by other scholars, which allowed us to compare results. Authors who have compared their data against the classic Ziegler-Nichols method have been selected.

For all cases, the program has been executed considering the following specifications: population size $=40$, number of variables $=3$, percentage of crossover $=70 \%$, percentage of mutation $=10 \%$, number of generations $=20$.

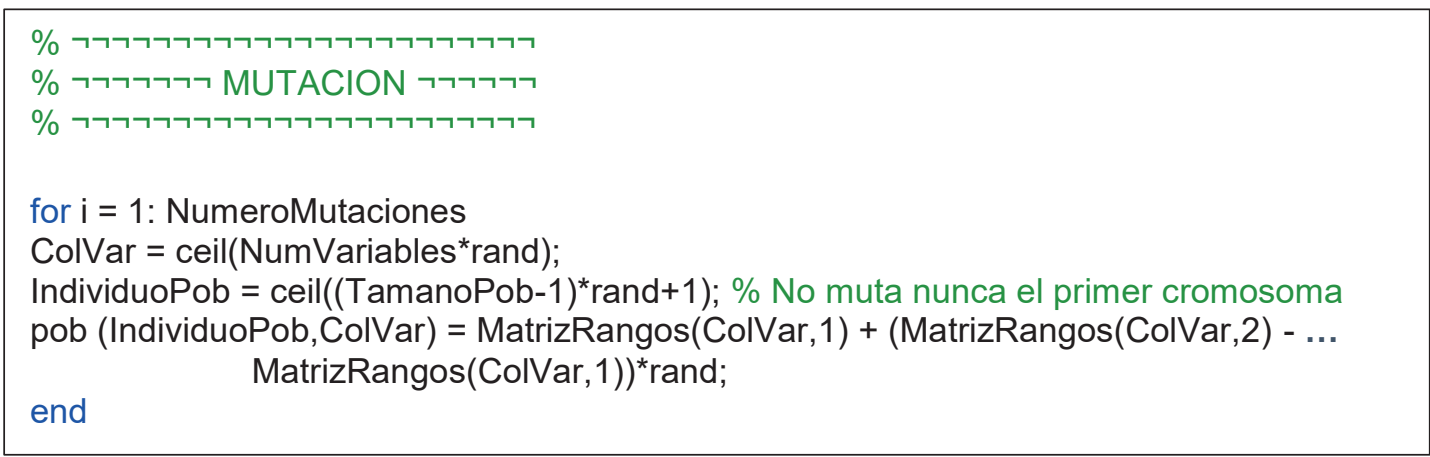

Figure 5. Portion of the mutation code.

Source: Prepared by the author. 


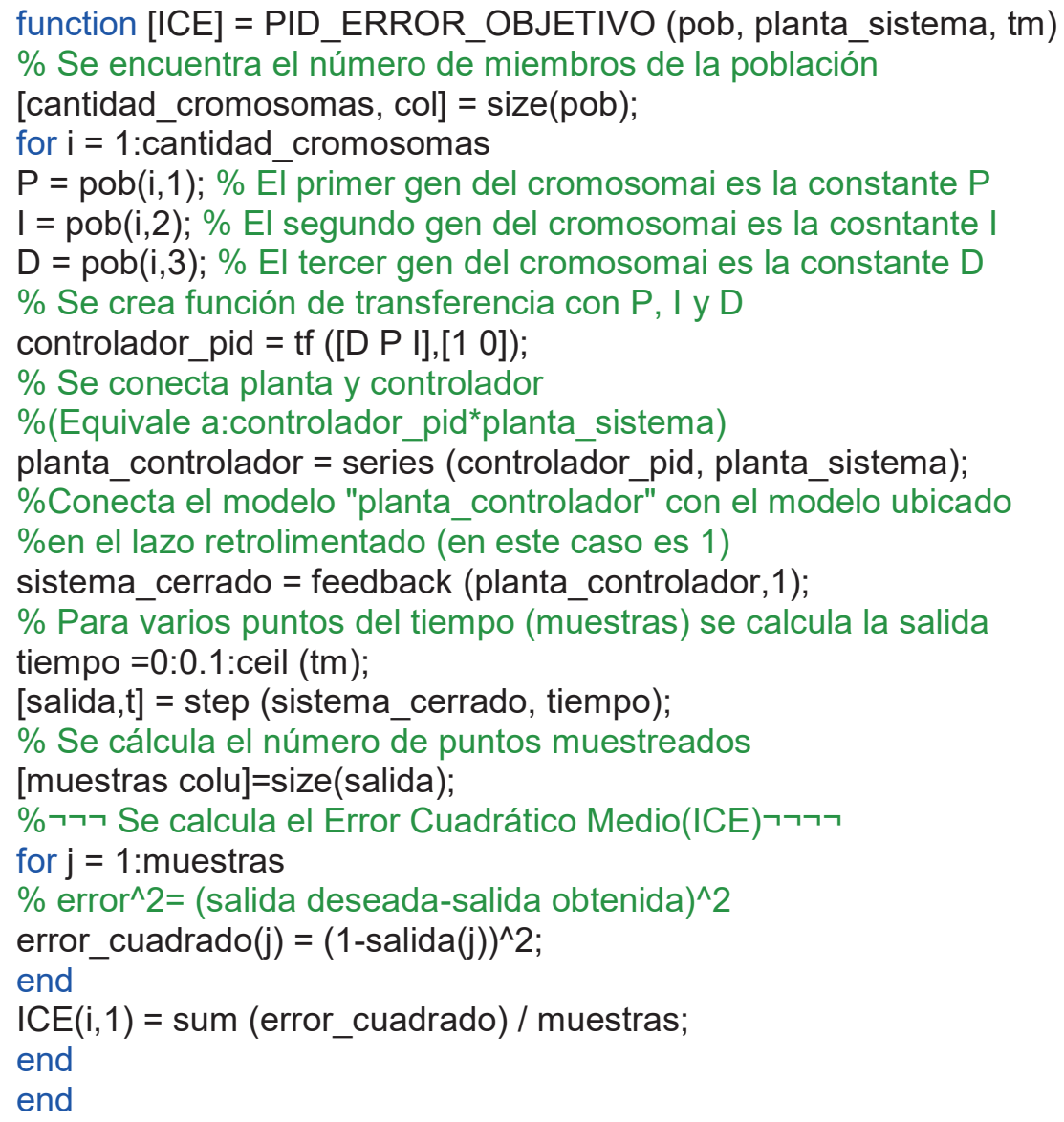

Figure 6. Evaluation code.

Source: Prepared by the author.

Tables 1-4 show the mathematical model of the controlled plant, the PID controller's parameters (Kp, $\mathrm{Ki}$ and $\mathrm{Kd}$ ), the output transient values (maximum overshoot, rise time, settling time) and the mean square error. In addition, the results are compared with those obtained by other researchers, and the column "Proposed genetic algorithm" corresponds to this study. The search intervals for each variable are displayed in the last row of each table.

Figures 7-10 graphically show the results of the above tables. The desired output of the system is shown with dotted lines and has been set to an amplitude of 1 (one step response). In addition, the blue line is the system output that finally reaches the desired output.

\section{DISCUSSION}

As can be seen from the results in Tables 1-4, in all cases maximum overshoot values were similar. Settling times were superior in all cases and rise time was best in all but Table 3. These results show that the proposed algorithm performed well in finding the appropriate $\mathrm{Kp}, \mathrm{Ki}$ and $\mathrm{Kd}$ values for the PID controller. In addition, the same specifications of population size, crossover percentage, mutation percentage and number of generations were used for all cases, demonstrating that the algorithm is quite robust.

\section{CONCLUSIONS}

Using genetic algorithms, the problem of tuning the proportional, derivative and integral parameters of a PID controller was solved, minimizing settling time and overshoot of the response in non-linear plants. It was also demonstrated that the results obtained were optimal when compared with the results of other investigations, shown in Tables 1-4.

In addition, it was demonstrated that the algorithm designed as a single-objective is efficient by indirectly minimizing various variables (overshoot, sett- 
Table 1. Comparison against results for Plant 1.

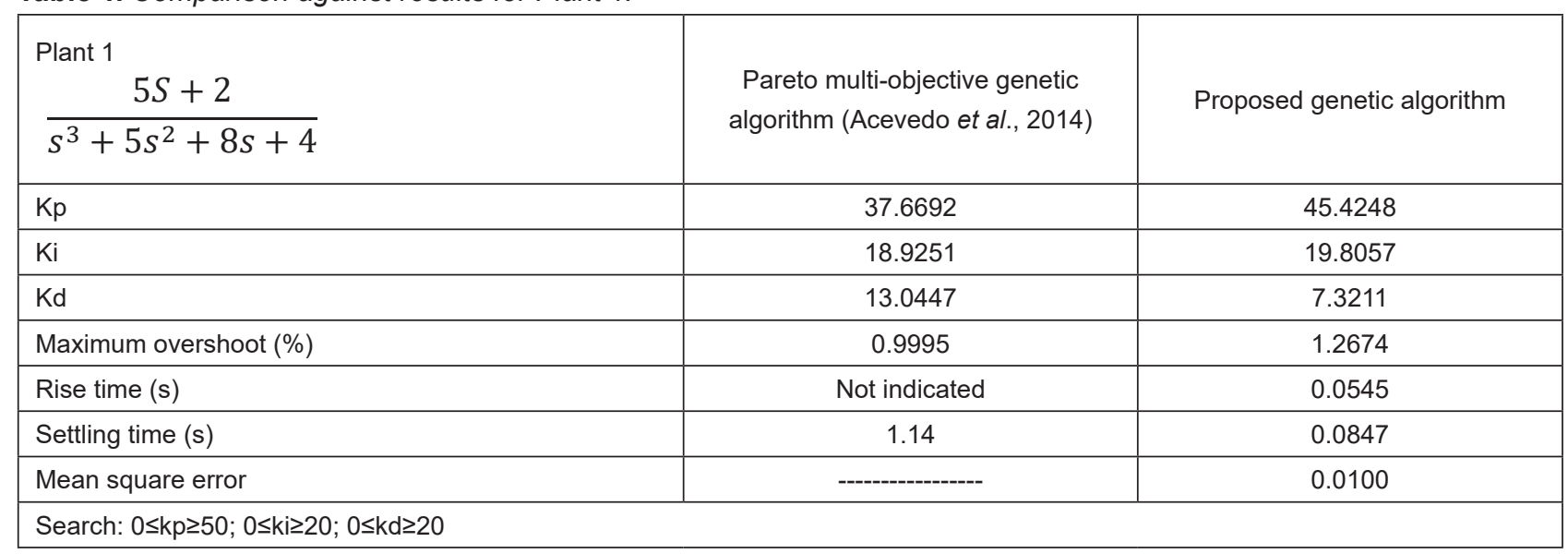

Source: Prepared by the author.

Table 2. Comparison against results for Plant 2.

\begin{tabular}{|c|c|c|}
\hline $\begin{array}{l}\text { Plant 2 } \\
\frac{10}{s^{2}+2 s+10}\end{array}$ & $\begin{array}{l}\text { Pareto multi-objective genetic } \\
\text { algorithm (Acevedo et al., 2014) }\end{array}$ & Proposed genetic algorithm \\
\hline Kp & 8.4047 & 48.1812 \\
\hline Ki & 17.1467 & 19.5887 \\
\hline $\mathrm{Kd}$ & 12.0918 & 11.6125 \\
\hline Maximum overshoot (\%) & 1.0137 & 1.1786 \\
\hline Rise time (s) & Not indicated & 0.0180 \\
\hline Settling time (s) & 1.9484 & 0.0291 \\
\hline Mean square error & ------------- & 0.0099 \\
\hline
\end{tabular}

Source: Prepared by the author.

Table 3. Comparison against results for Plant 3.

\begin{tabular}{|c|c|c|}
\hline $\begin{array}{l}\text { Plant 3. Control of three tanks' level } \\
\frac{1}{64 s^{3}+9,6 s^{2}+0,48 s+0,008}\end{array}$ & $\begin{array}{l}\text { Genetic algorithm } \\
\text { (Colorado et al., 2018) }\end{array}$ & Proposed genetic algorithm \\
\hline Kp & 0.0361 & 0.03827 \\
\hline Ki & 0.000731 & 0.00061 \\
\hline $\mathrm{Kd}$ & 0.6999 & 0.63012 \\
\hline Maximum overshoot (\%) & 18 & 12.3728 \\
\hline Rise time (sec.) & 10 & 16.3166 \\
\hline Settling time (sec.) & 90 & 86.0968 \\
\hline Peak time & 45 & 33.5201 \\
\hline Mean square error & --------- & 0.03280 \\
\hline
\end{tabular}

Source: Prepared by the author. 
Table 4. Comparison against results for Plant 4.

\begin{tabular}{|l|l|l|}
\hline $\begin{array}{l}\text { Plant 4. Control of tank level } \\
\frac{5(s+1)}{S(s+1)(s+6)}=\frac{5 s+5}{s^{3}+7 s^{2}+6 s}\end{array}$ & $\begin{array}{l}\text { Fuzzy PD+PID Controller (Souran et } \\
\text { al., 2013) }\end{array}$ & Proposed genetic algorithm \\
\hline $\mathrm{Kp}$ & 30 & 39.10042 \\
\hline $\mathrm{Ki}$ & 21.2 & 1.16204 \\
\hline $\mathrm{Kd}$ & 9 & 6.86381 \\
\hline Maximum overshoot $(\%)$ & 0.72 & 0 \\
\hline Rise time $($ sec.) & Not indicated & 0.06548 \\
\hline Settling time $($ sec.) & 23 & 0.12271 \\
\hline Mean square error & & 0.01236 \\
\hline Search: $0 \leq \mathrm{kp} \geq 40 ; 0 \leq \mathrm{ki} \geq 30 ; 0 \leq \mathrm{kd} \geq 20$ & \multicolumn{2}{l}{} \\
\hline
\end{tabular}

Source: Prepared by the author.

Step Res porse

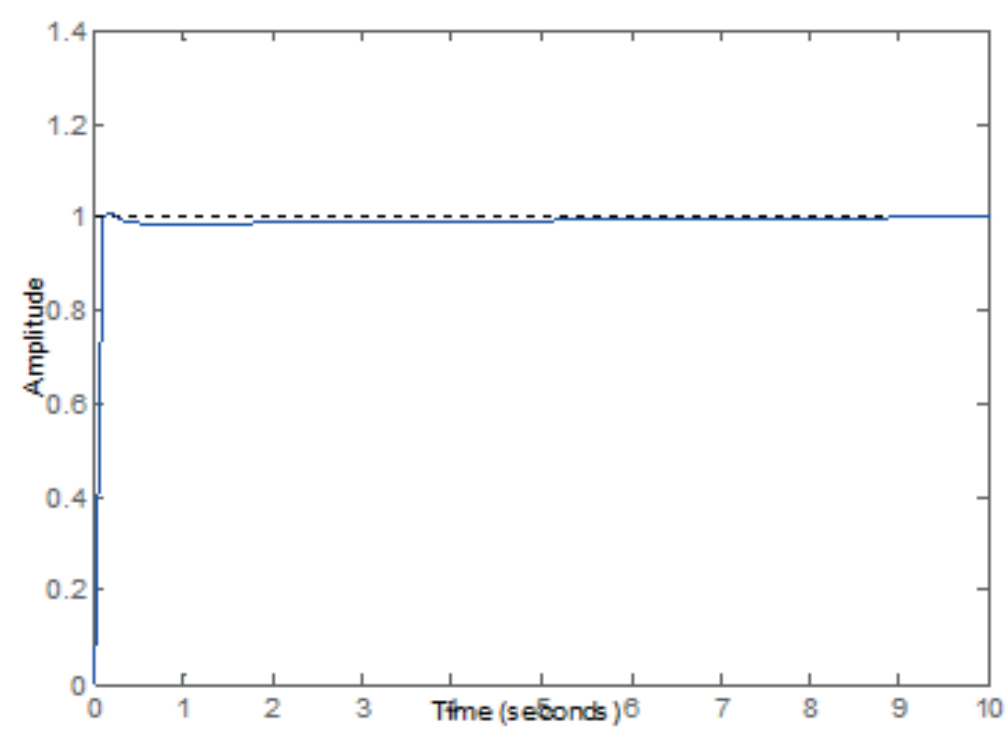

Figure 7. Controlled response from Plant 1 (Table 1).

Source: Prepared by the author.

ling time, rise time) by minimizing a single variable: mean square error, as opposed to a multi-objective genetic algorithm based on the Pareto approach, as presented by Acevedo et al. (2014).

\section{REFERENCES}

[1] Acevedo, B.; Fonseca, J. y Gómez, J. (2014). Desarrollo de una herramienta en Matlab para sintonización de controladores PID, utilizando algoritmos genéticos basado en técnicas de optimización multiobjetivo. Revista SENNOVA, 1(1), 80-103.
[2] Akbari-Hasanjani, R.; Javadi, S. y SabbaghiNadooshan, R. (2015). DC motor speed control by self-turning fuzzy PID algorithm. Transactions of the Institute of Measurement and Control, 37(2), 164-176.

[3] Beck, F. (2000). Escalonamiento de tarefas jobshop realistas utilizando algoritmos genéticos en Matlab. (Tesis de maestría). Universidad Federal de Santa Catarina, Florianópolis, SC.

[4] Colorado, O.; Hernández, N.; Seck Tuoh, J. y Medina, J. (2018). Algoritmo genético aplicado a la sintonización de un controlador PID para 
Step Response

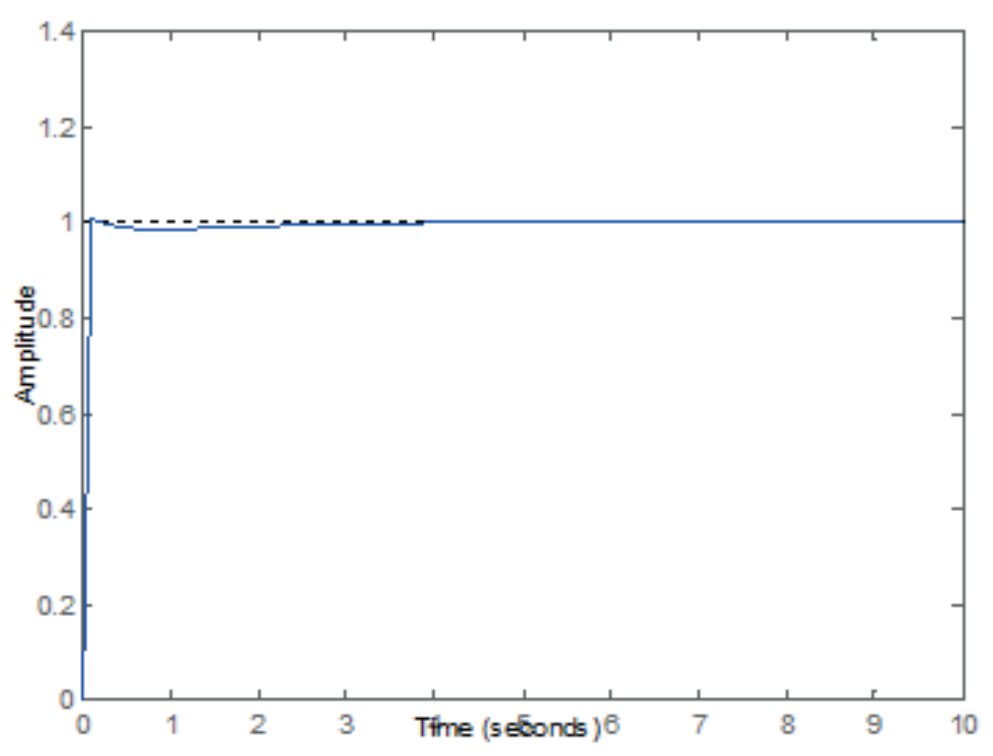

Figure 8. Controlled response from Plant 2 (Table 2). Source: Prepared by the author.

Step Res porse

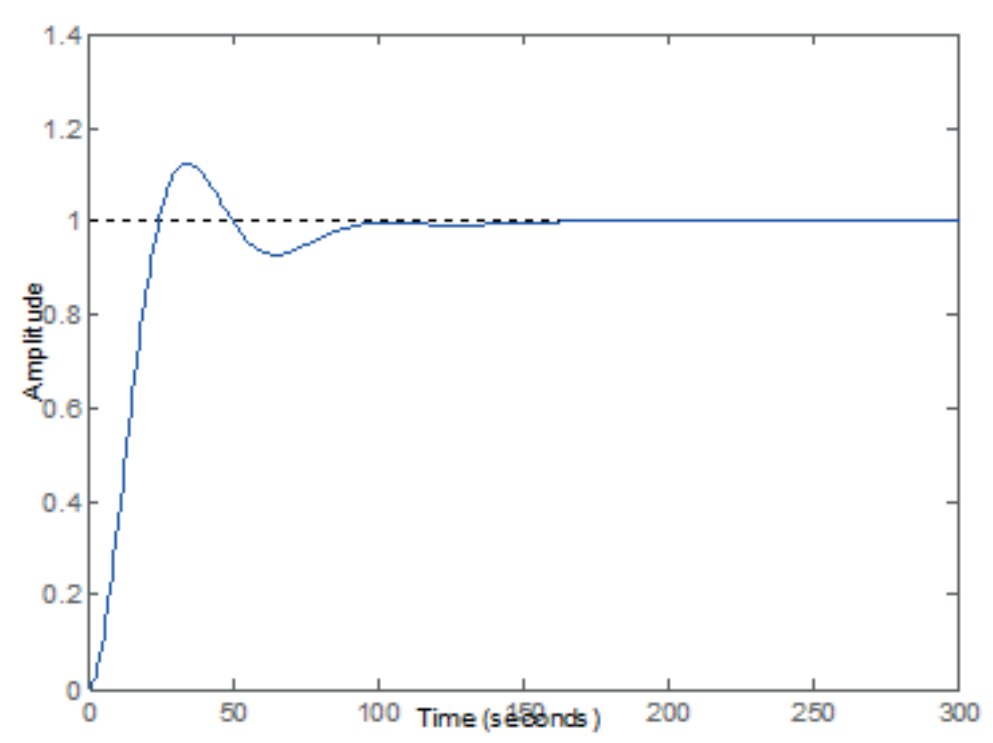

Figure 9. Controlled response from Plant 3 (Table 3).

Source: Prepared by the author. 


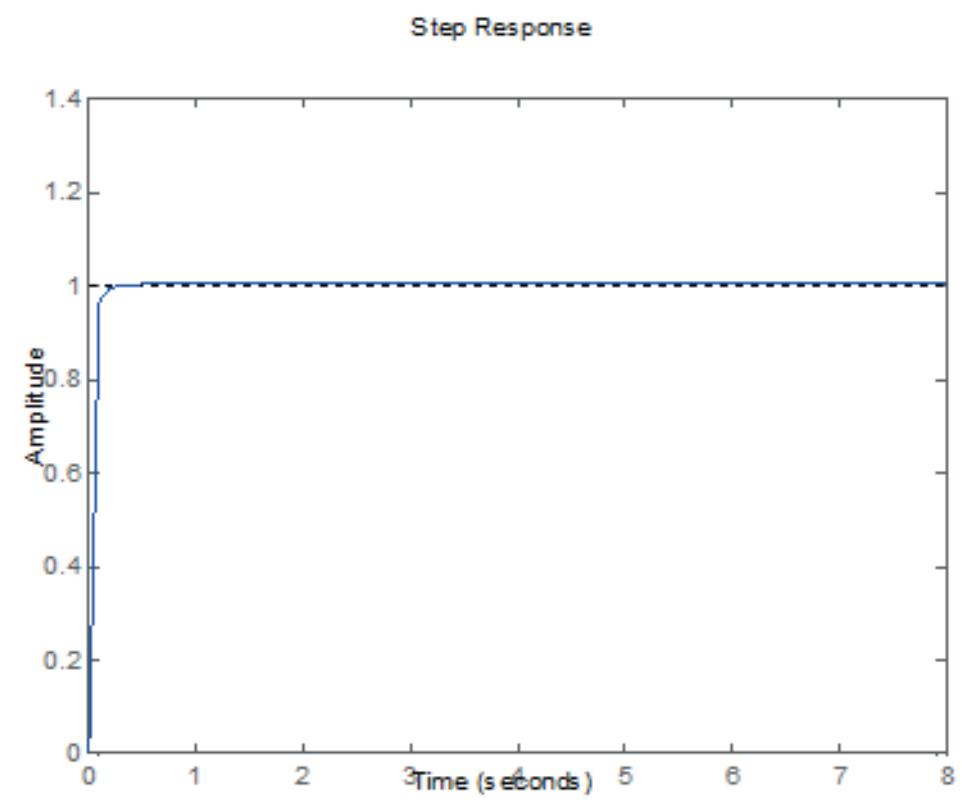

Figure 10. Controlled response from Plant 4 (Table 4).

Source: Prepared by the author.

un sistema acoplado de tanques. PADI Boletín Científico de Ciencias Básicas e Ingenierías del ICBI, 5(10), 50-55.

[5] Feng, H.; Yin, C.; Weng, W.; Ma, W.; Zhou, J.; Jia, W. y Zhang, Z. (2018). Robotic excavator trajectory control using an improved GA based PID controller. Mechanical Systems and Signal Processing, 105, 153-168.

[6] Gestal, M.; Rivero, D.; Rabuñal, J.; Dorado, J. y Pazos, A. (2010). Introducción a los algoritmos genéticos y la programación genética. La Coruña, España: Universidad de La Coruña.

[7] Haupt, R. y Haupt, S. (2004). Practical genetic algorithms. Nueva Jersey, EE. UU.: WileyInterscience.

[8] Hernández, R.; García, L.; Salgado, T.; Gómez, A. y Fonseca, F. (2016). Neural networkbased self-turning PID control for underwater vehicles. Sensors, 16(9). Recuperado de https://www.ncbi.nlm.nih.gov/pmc/articles/ PMC5038707/.

[9] Iwan, M.; Fook, L. y Leap, M. (2011). Tuning of PID controller using particle swarm optimization (PSO). International Journal on Advanced Science, Engineering and Information Technology, 1(4), 458-461. Recuperado de http://insightsociety.org/ojaseit/index.php/ ijaseit/article/viewFile/93/98.
[10] Lahlouh, I.; Elakkary, A. y Sefiani, N. (2019). PID controller of a MIMO system using ant colony algorithm and its application to a poultry house system. En H. Hachimi (Ed.), 2019 5th International Conference on Optimization and Application (ICOA) llevado a cabo en Marruecos.

[11] Mohamed, I. (2005). The PID controller design using genetic algorithm. (Tesis de pregrado). University of Southern Queensland, Toowoomba, QLD.

[12] Pezzella, F.; Morganti, G. y Ciaschetti, G. (2008). A genetic algorithm for the flexible job-shop scheduling problema. Computers \& Operations Research, 35(10), 3202-3212.

[13] Schaffer, J. y Eshelman, L. (1991). On crossover as an evolutionarily viable strategy. International Conference on Genetic Algorithms, 4, 61-68.

[14] Seck Tuoh, J.; Medina, J. y Hernández, N. (2016). Introducción a los algoritmos genéticos con Matlab. Recuperado de https:// repository.uaeh.edu.mx/bitstream/bitstream/ handle/123456789/16991/introduccion_a_los_ algoritmos_geneticos_con_matlab.pdf.

[15] Souran, D.; Abbasi, S. y Shabaninia, F. (2013). Comparative study between tank's water level control using PID and fuzzy logic controller. En V. Balas, J. Fodor, A. Várkonyi-Kóczy, J. Dombi 
y L. Jain (Eds.), Soft computing applications $V$. 195 (pp. 141-153). Berlín, Alemania: SpringerVerlag Berlin Heidelberg.

[16] Srinivas, M. y Patnaik, L. (1994). Adaptive probabilities of crossover and mutation in genetic algorithms. IEEE Transactions on Systems, Man, and Cybernetics, 24(4), 656667.
[17] Tejada, G. (2017). Enrutamiento y secuenciación óptimos en un flexible job shop multiobjetivo mediante algoritmos genéticos. (Tesis doctoral). Universidad Nacional Mayor de San Marcos, Lima.

[18] Yalcinoz, T. y Altun, H. (2001). Power economic dispatch using a hybrid genetic algorithm. IEEE Power Engineering Review, 21(3), 59-60. 